einem Kraftverbrauch von $3 \mathrm{HP}$. am Generator, gebohrt, später stieg der Effekt auf 4 Zoll per Minute. Die Maschine soll sich wenig erhitzen.

Nach anderer Mitteilung soll eine solche Bohrmaschine bei 650 Schlägen per Minute $5 \mathrm{HP}$. erfordern, 4 Zoll lange Schläge machen und 8 Fuss ein - bis zweizöllige Löcher im Granit bohren. Anderseits behauptet man, dass so Ieichte Schläge, wie zum Abbohren eines Bohrloches erforderlich, damit erfolgen können, was ein vorteilhafter Umstand wäre. Nach dem Gesagten sollte man annehmen, dass elektrisch betriebene Bohrmaschinen bereits allgemeinere Anwendung in den VereinigtenStaaten von Nordamerika gefunden hätten und dass man da-

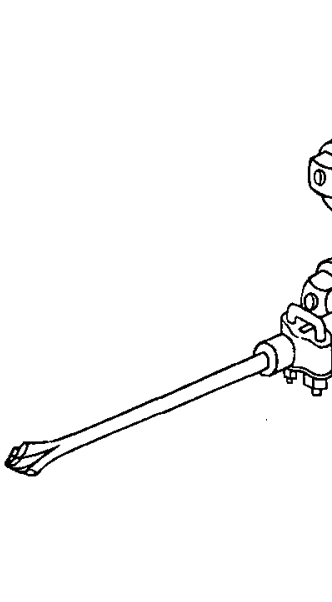
mit recht vorteilhaft arbeite; nach privaten aber, wie auch im Druck veröffentlichten Mitteilungen aus Amerika scheint man aber doch sich damit noch im Versuchsstadium zu befinden. Verschiedene elektrische Bohrmaschinen-Anordnungen sind allerdings ausgeführt, aber ganz zufrieden mit den erreichten Resultaten scheint man doch noch nicht zu sein.

Das Engineering and Mining Journal sagt im
Januar I 892 auch, dass, obschon die Einfachheit der elektrischen Stossbohrer sich empfiehlt, doch die Erfahrung noch nicht ergeben hat, dass das Solenoidprinzip ohne weitere Verbesserung für das Bergbohren mit gleichem Erfolge und gleicher Ökonomie angewendet werden kann, welche man beim Bohren mit Dampf oder Pressluft erreicht. Die Hitze innerhalb der Maschine beim Betriebe ist nicht allein eine wirkliche Unannehmlichkeit, sondern auch eine Quelle von Kraftverlust; andere Schwierigkeiten erwachsen aus der magnetischenBeeinflussung der Hubstange, der Bohrstange und anderer Teile, die nicht allein aus einzelnen Stücken, sondern auch aus nicht magnetischem Metall ge-

ig. 21 . fertigt werden müssen. Eine weitere Beeinträchtigung erwächst aus dem schweren Gewichte des Bohrsystems. Bei den elektrischen Maschinen ist der Effekt dem Gewichte proportional, welches nicht der Fall ist bei Dampf- und Pressluftbohrmaschinen, welche im Verhältnis zum Gewicht einen grösseren Effekt geben, etwa I HP. für ungefähr $22,25 \mathrm{~kg}$ ihres Gewichtes.
(Fortsetzung folgt.)

\title{
KLEINS VERFAHREN ZUM VERDICHTEN UND FORMEN ELEKTROLYTISCHER METALLNIEDERSCHLÄGE.
}

Von Dr. G. Langbein.

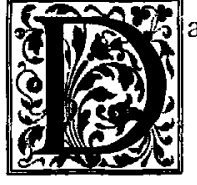
allgemeine Interesse, welches auf der mit dem Verbandstage deutscher Elektrotechniker verbundenen Ausstellung den von mir vorgeführten Kleinschen Modellen zur Herstellung von Kupferröhren, profilierten Hohlkörpern etc. entgegengebracht wurde, giebt mir die Überzeugung, dass eine eingehendere Beschreibung dieses Verfahrens besonders für den Leserkreis dieser Zeitschrift von Interesse sein dürfte.

Die vorliegende Erfindung betrifft das Walken des sich infolge galvanischen Prozesses niederschlagenden Elektrolytmetalles zum Zwecke des Ver- dichtens und Formens desselben. Das Walken ist dadurch gekennzeichnet, dass walzenartige Kathoden, auf welchen sich Elektrolytmetall niederschlagen soll und welche ein beliebiges Längsprofil haben können, während der Bildung des Niederschlages auf geraden oder gebogenen Platten mit dem gleichen Längsprofil, sogenannten Walkplatten, unter entsprechendem Druck gewälat werden. Infolge dieser Prozedur wird der Niederschlag die Gestalt von hohlen Drehkörpern, deren Längsprofil demjenigen der Kathoden und der Walkplatten entspricht, annehmen und einen höheren Grad von Zăhigkeit erlangen; 
dabei gestattet die Konstruktion der Walkapparate die gleichzeitige Verwendung mehrerer, auch verschieden profilierter Walkplatten in ein und demselben elektrolytischen Bade; die Anzahl der gleichzeitig herzustellenden Drehkörper hingegen hängt von der Dimension der Walkvorrichtung ab.

Im wesentlichen sind zwei Sorten dieser Drehhohlkörper zu unterscheiden, nämlich solche, welche als selbständige Hohlkörper und solche, welche als bleibende metallische Überzüge auf den ursprünglichen Niederschlagsformen Verwendung finden.

Für beide Sorten besteht die Niederschlagsform, kurzweg Kern genannt, wesentlich aus einem festen Gerippe, welches mit einem leicht schmelzbaren Überzuge versehen ist.

Die Kerngerippe selbst bestehen aus widerstandsfähigem Stoffe, wie Metall, Holz u. dgl. und sind

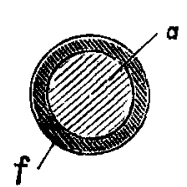

Fig. I.

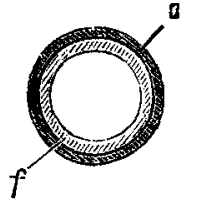

Fig. 2.

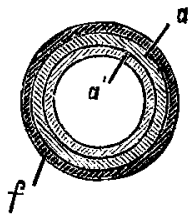

Fig. 3.

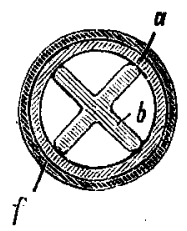

Fig. 4. entweder voll $a$ (Fig. I) oder hohl (Fig. 2). Lange hohle Kerne werclen durch ein eingeschobenes Rohr $a^{1}$ (Fig. 3) oder ein passendes Kreuzstück (Fig. 4) versteift.

Die Enden hohler Kerne werden durch mit Drehzapfen $d$ (Fig. 5) und metallischen Kontaktkappen $\varepsilon$. versehene Spunde $c$ dicht geschlossen. Je nachdem die Drehhohlkörper der einen oder anderen Sorte angehören, werden die Kerngerippe entweder vorerst mit einem entsprechenden plastischen Stoffe wie Gips, Kitt, Thon, Maché u. dgl. umhüllt oder auch unmittelbar mit einer Schicht $f$ eines leicht schmelzbaren, polierbaren und von der Flüssigkeit im Bade unangreifbaren Stoffes, wie Blei
Zur massenhaften und präzisen Herstellung der Kerne verwendet man mit Vorteil die in Fig. 6 dargestellte Vorrichtung, bestehend aus zwei auf dem Postamente $k$ gerau parallel befestigten Laufichienen $a$, zwischen welchen die Walkplatte $c$ liegt, ferner aus den zu jedem Kerngerippe $d$ gehörigen, mit je einer Leitrolle $g$, Gleitmuffen $h$ und Einstellschraube $b$ montierten Spindelpaaren $j j$. Vorerst wird auf den Kerngerippen $d$ die Grundform $e$ aus Thon u. dgl. durch Hin- und Herrollen auf der Walkplatte hergestellt, hart gelrannt und mit einer Fettschicht umhüllt; sodlann werden die Ringe $i$ auf die Muffen $h$ gesteckt und die Kerne mittels eines Rahmens bis zur Fertigstellung auf derselben Walkplatte hin- und hergerollt. Durch Anwendung mehrerer, der Reihe nach stärkeren Ringe $i$ kann das Gerippe auch mit mehreren konzentrischen Schichten umhüllt werden. Derart hergestellte Kerne bilden die walzenartigen Kathoden.

Die Vorrichtungen, welche zum Walken dienen, sind in ihren wichtigsten Ausführungsformen auf den nachstehenden Figuren dargestellt.

Die einfachste Ausführungsform der Walkvorrichtung zeigen Fig. 7 und 8 .

In einem das elelstrolytische Bad aufnchmenden Trog $A$ ruht auf Unterlagen $g$ die Walkplatte $h$, welche aus Glas, Porzellan oder einem ähnlichen harten, von dem Bade unangreifbaren Stoffe besteht. Auf die Walkplatte wird eine Anzahl Kerne gelegt, welche in einem Rahmen $i$ parallel und clerart cingesetzt sind, dass ihre Drehzapfen $d$ in nach unten offenen Schlitzen zweier gegenüber licgender Leisten des Rahmens lagern, so dass der Rahmen, welcher auf den Drehzapfen frei aufliegt, von den Kernen leicht alugehoben werclen kann. Die Zuleitung des elektrischen Stromes vom negativen Pole der Elektricitätsquelle zu den leitend gemachten Oberflächen der Kerne erfolgt mittels Drahtes $m^{1}$, der am

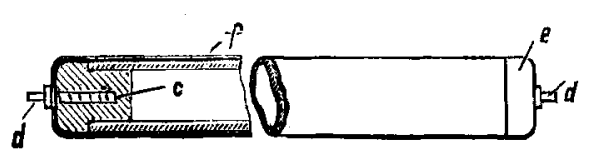

Fig. 5 .

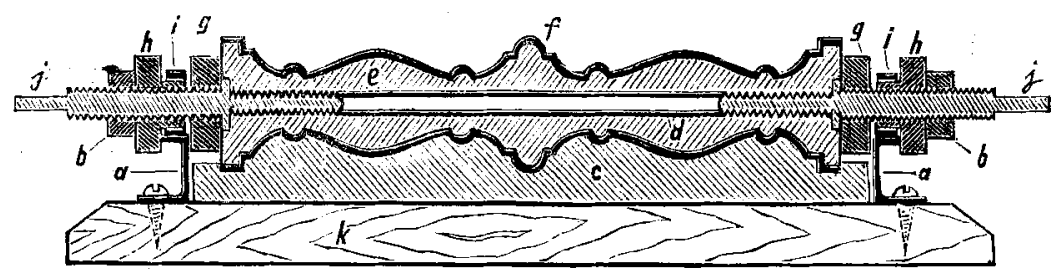

Fig. 6.

und dessen Legierungen, ferner Lack, Wachs, Stearin, Paraffin $u$. dgl. oder deren Gemenge überzogen, in die den darzustellenden Gegenstünden entsprechende Form gebracht und an ihrer Aussenflache leitend gemacht.
Rahmen montierten Lamellen oder Bürsten $L$ und der auf die Kernenden gesetzten Kontaktkappen $\boldsymbol{c}$. Parallel zur Mantelfäche der Kerne befindet sich eine Anodenplatte, mit dem positiven Pole der Elektricitätsquelle durch den Draht $m$ verbunden. 
Sobald der Walktrog mit der entsprechenden Flüssigkeit gefüllt und der elektrische Strom ge-

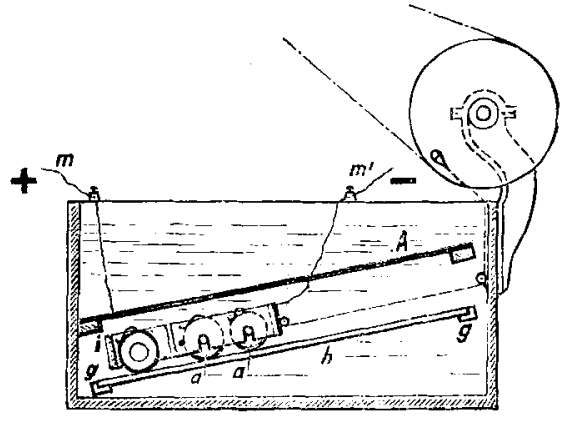

Fig. 7 .

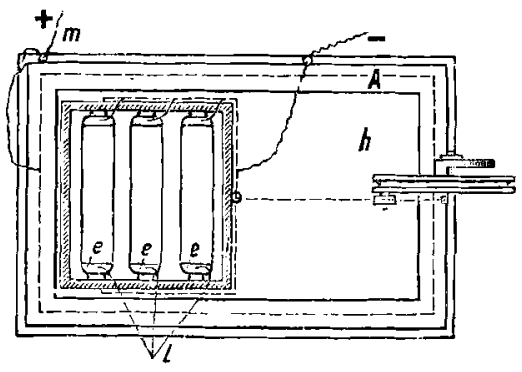

Fig. 8. cylindrischen Behälters $a$ für die Flüssigkeit befestigt ist. $d$ ist das in dem Behälter $a$ gelagerte Dreh- schlossen ist, wird der frei aufliegende oder belastete oder sonst unter Druck stehende Rahmen zwischen Anode und Walkplatte so lange hin- und hergezogen, bis sich auf den Kernen, welche sich bei dieser Prozedur um das Mehrfache ihres Umfanges abwälzen müssen, der Niederschlag in der erforderlichen Stärke gebildet hat. Hierbei kann die Walkplatte $h$ horizontal oder unter beliebigem Winkel geneigt liegen und die Anzahl und Form der auf einmal zu walkenden Kerne beliebig sein, sofern die Walkplatte $h$ genügend gross ist und ein der Form der Kerne entsprechendes Querprofil besitzt (Fig. 9 u. Io).

Fig. I I zeirgt eine Ausfülrungsform, welche als ein Mehrfaches der in Fig. 7 und 8 dargestellten betrachtet werden kann.

Fig. I 2 und I 3 zeigen den Lüngs-bezw. Querschnitt eines Walktroges, in welchem das Walken beliebig vieler Reihen von Kernen beliebigen Querprofiles in senkrechter Richtung erfolgt, wobei die Geradführung des Rahmens durch Gleitrollen $r^{1}$ (Fig. I2) und das Andrücken cler Kerne an die Walkplatten mittels elastischer Pressrollen $r^{2}$ geschieht. Das Aupressen des Rahmens kann auch mittels einstellbarer Keile oder auf ähnliche Weise erfolgen.

Die den Erhabenheiten des Kernes entsprechend gebugene Anodenplatte kann in der erforderlichen Weise auch auf den Rahmen montiert werden und an der Bewegung des letzteren teilnehmen (Fig. I 3).

Die Walkplatte kann statt gerade auch muldenförmig gekrümmt sein; in diesem Falle ist dem die Kerne führonden Rahmen cine entsprechend gebogene Form zu geben.

Ferner kann man die Walkplatte zu einem Hohlcylinder und den Rahmen zu einem Drehgestelle ausbilden, wie dies Fig: I 4 im Querschnitt zeigt. In dieser ist $/$ der Walkcylinder, welcher mittels seiner Einfassung $c$ an den Stirnwänden des halb- gestelle, welches die in Cylinderform gebogene Anode $f$ und zwei Scheiben $e$, an jedem Ende eine,

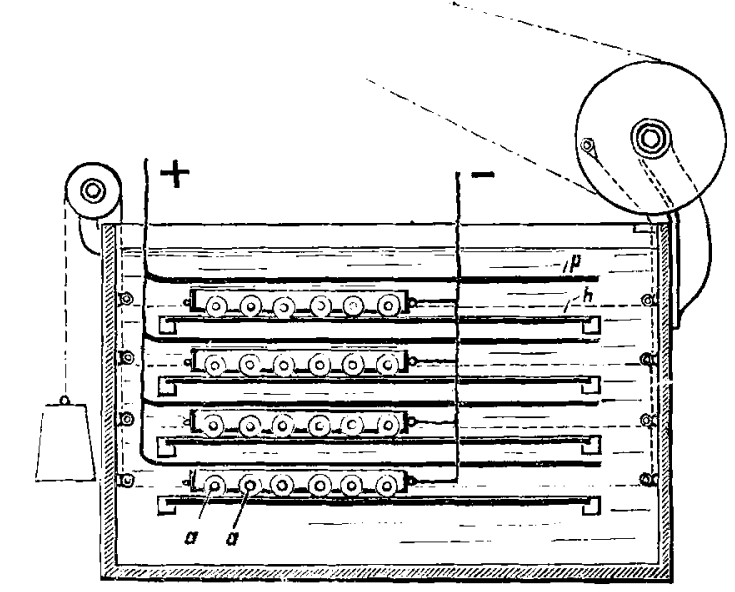

Fig. 12 .

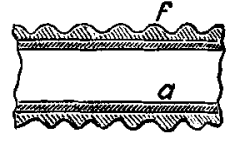

Fig. 9.

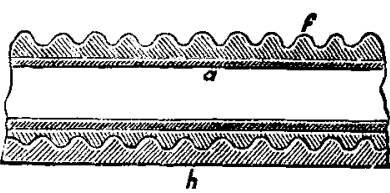

Fig. 10 .
Fig. I I.

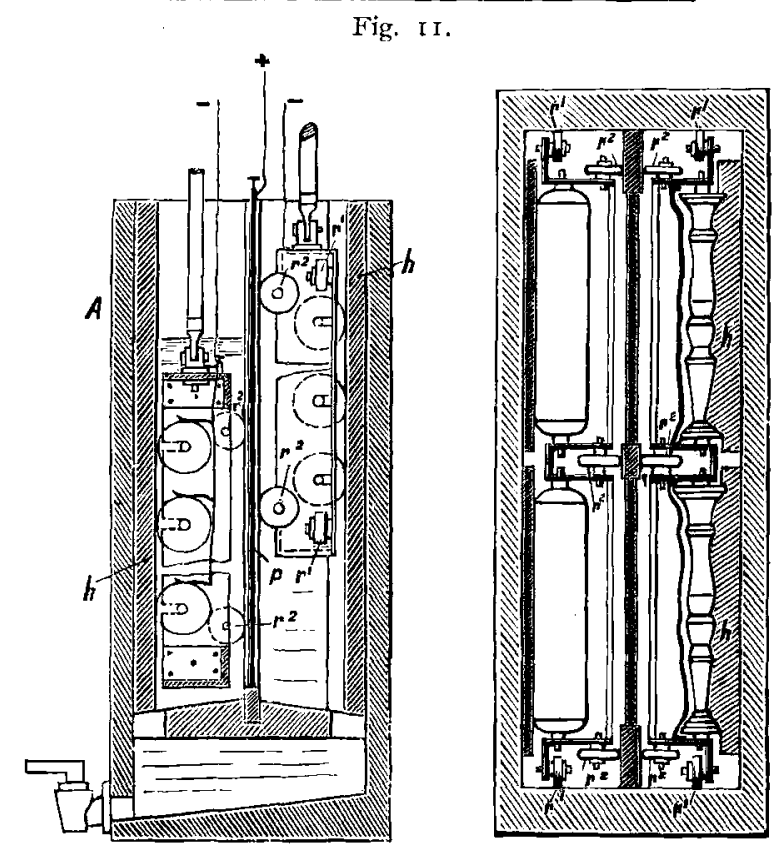

Fig. 13. trägt, in denen in nach aussen offenen Schlitzen die Zap̣fen der Kerne lagern. Bewegt man das 
Drehgestelle $d$ abwechselnd in dem einen und dem andern, oder stets in demselben Sinne, wobei die Kerne an den Walkcylinder durch die auftre-

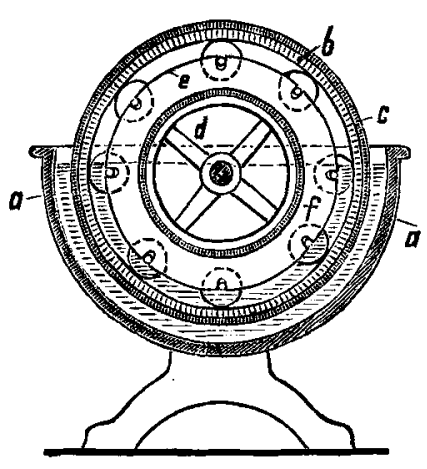

Fig. I4.
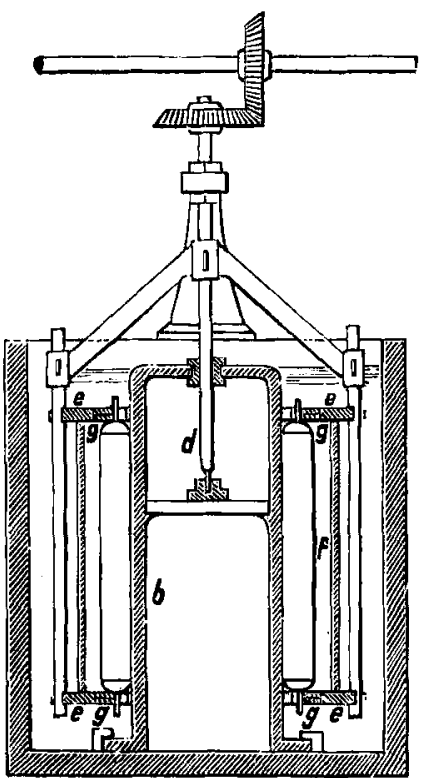

Fig. I 5 . tende Centrifugalkraft oder in sonst geeigneter Weise angedrückt werden, so wird der auf den Kernen abgelagerte Niederschlag in derselben Weise, wie bei den zuvor beschriebenen Vorrichtungen verdichtet. Selbstverständlich kann die Vorrichtung auch in vertikaler Stellung arbeitend konstruiert werden. Eine ăhnliche Ausführungsform wie Fig. I4 zeigt Fig. I5; hier ist der Walkcylinder senkrecht gestellt und in der Mitte des Troges befestigt, wăhrend die in einem Drehgestelle montierten Kerne auf der Aussenflache des Waikcylinders abgewälzt werden, wobei sie etwa durch Federn an den Cylinder angedrückt erhalten bleiben.

Bei der in Fig. I6 dargestellten Ausführungsform bildet die Führung für die Kerne eine Drehscheibe, zu deren Drehachse die Keme radial liegen. Es können mehrere solcher Vorrichtungen etagenartig übereinander angeordnet und deren Drehscheiben an einer gemeinschaftlichen Mittelwelle befestigt werden.

In Fig. 17 ist das Walken des Niederschlages auf der Innen- und Aussenfläche zweier Hohlkerne innerlich durch Walkwalzen w, tusserlich durch die Walkplatte $p$ dargestellt, wobei die inneren Anoden mit $a$, die äusseren mit $b$ bezeichnet sind.

Es ist ratsam, bei all diesen Vorrichtungen anfänglich mässige Geschwindigkeit und geringeren Druck anzuwenden.

Der durch das Walken gekennzeichnete Arbeits- vorgang kann bei Ersatz der Walkplatten oder Cylinder durch entsprechende Poliervorrichtungen auch in der Galvanostegie (Galvanisches Vernickeln, Versilbern etc.) Anwendung finden.

Gegenüber dem in Schladern in Ausübung befindlichen Elmore-Verfahren hat das Kleinsche so entschiedene Vorzüge, dass eine Rentabilităt des Grossbetriebes gesichert zu sein scheint, was bei

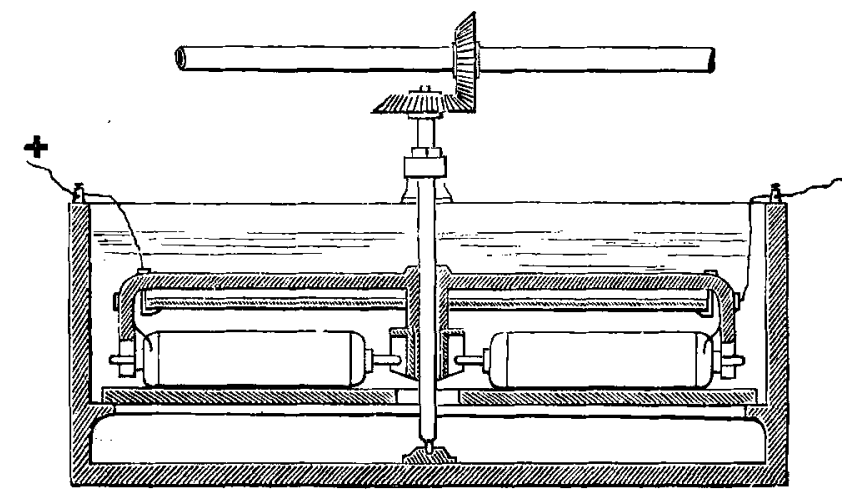

Fig. I 6.

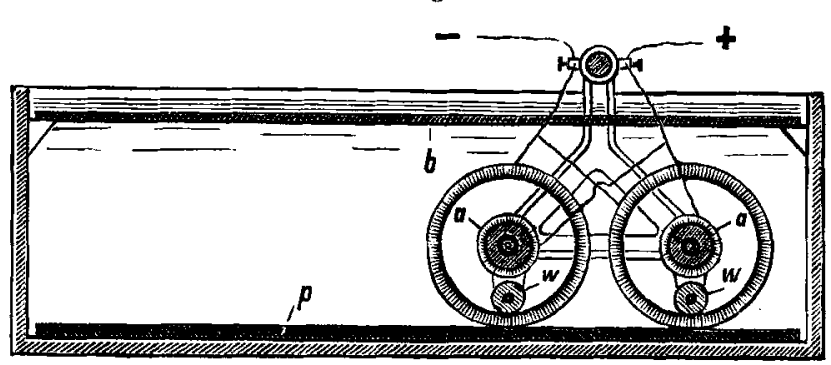

Fig. 17.

dem Elmore-Verfahren bis jetzt noch zweifelhaft sein soll. Elmore braucht, da er wegen der Vorrichtungen für die, die Dichtung der Niederschläge bewirkenden Achate den Bäderraum nicht gut ausnutzen kann, eine relativ grosse Menge Bäder, demnach viel Raum, beträchtliche Vorräte von Anoden und Elektrolyt-Substanz, während beim Kleinschen Verfahren in Bädern gleicher Grösse ungleich grössere Warenmengen erzeugt werden können, wodurch sich Amortisation und Zinsen auf die Einheit des Niederschlages wesentlich günstiger stellen. Nach dem Elmore-Verfahren können bekanntlich nur glatte cylindrische Objekte erhalten werden; das Kleinsche Verfahren dagegen gestattet die Herstellung profilierter Rundkörper, z. B. Ballustradesäulen u. dgl. mit grösster Leichtigkeit. Wie die nach diesem Verfahren hergestellten Proben ergeben haben, ist das Kupfer ausserdem von ausgezeichneter Beschaffenheit, sehr zäh und sehr widerstandsfähig. Eine Anwendung des Kleinschen Verfahrens, die eine bedeutende Zukunft haben dürfte, will ich nur noch 
erwähnen, nämlich die Herstellung gewellter Siederöhren für Dampfkessel, besonders von Lokomotiven, in denen die Vergrösserung der Heizfläche von Wichtigkeit ist. Interessenten stehe ich gern mit näheren Angaben zur Verfügung.

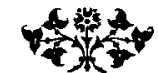

\section{ELEKTRISCHER BAHNBETRIEB MITTELS TRANSPORTABLER AKKU. MULATOREN.}

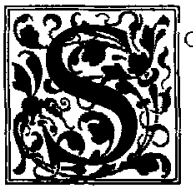

lange es elektrische Bahnen giebt, so lange ist die Akkumulatorenfrage noch nicht von der Reihe der brennenden Tagesfragen gewichen.

Man kennt die Vorzüge dieses Betriebes vom Verkehrs-Standpunkte aus zu gut, als dass es nötig wäre, an dieser Stelle auf die Einzelheiten näher einzugehen; man weiss aber auch, dass sich bisher unüberwindliche wirtschaftliche Schwierigkeiten gezeigt haben und dass bisher noch kein Unternehmer den Mut gehabt hat, aus dem Stadium des Versuches in die Praxis hinauszutreten. Durch Wort und Schrift wird wahrlich genügend agitiert, denn dadurch übernimmt niemand ein Risiko. Den Beweis durch die Thatsache anzutreten, hat aber noch niemand versucht.

Man darf freilich deshalb nicht achtlos an der Litteratur vorübergehen und jedes rechnerisch unanfechtbar durchgeführte Beispiel ist eines năheren Eingehens wert. Vor uns liegt ein Aufsatz von einem Akkumulatoren-Techniker (L. Epstein), welcher in der Electrical Review am 5. Januar I 894 erschienen ist, und welcher in den rosigsten Hoffnungen ausklingt. Der Verfasser hat sich die Mühe genommen, Vergleiche mit bestehenden elektrischen Betrieben des oberirdischen und unterirdischen direkten Stromzuführungssystems anzustellen.

$\mathrm{Da}$ es schwer ist, derartige allgemeine Vergleiche auf ihre Richtigkeit zu prüfen, weil die örtlichen etc. Verhältnisse stets dem einen oder anderen System günstig sein werden, soll hier versucht werden, das Nachfolgende auf seinen absoluten Wert zu prüfen und dazu ist es nötig, dass die Behauptungen zur allgemeinen Kenntnis und Diskussion in deutscher Sprache gebracht werden.

Es möge daher zunächst die abgekürzte Übersetzung des englischen Aufsatzes mit den für unsere Verhältnisse umgerechneten Tabellen folgen.
Unter dem Titel:

"Mehr Licht über elektrische Zugkraft"“

schreibt L. Epstein:

Die Tage der tierischen Zugkraft für Bahnen sind gezählt und an deren Stelle muss motorische Kraft in der einen oder anderen Form treten.

Von einem Vergleich der verschiedenen motorischen Systeme wie: Dampf-, Kabel-, elektrischen Bahn-System, wird hier abgesehen.

Der Zweck des folgenden Aufsatzes ist, die speziellen Vorteile und die Betriebskosten der verschiedenen elektrischen Systeme miteinander zu vergleichen und zwar unter Zugrundelegung praktischer Resultate, die einigen im Betrieb befindlichen Bahnen entnommen sind.

Der Verfasser würde für jede ergänzende Belehrung dankbar sein, sofern sie den Gegenstand betrifft und aus praktischen Versuchen und Vergleichen stammt.

Im Folgenden bedeutet:

$O=$ oberirdisches Stromzuführungs -System,

$U=$ unterirdisches " "

$A=$ Akkumulatoren - System.

\section{Die Kosten der Schienen}

sind für alle 3 Systeme gleich und betragen für das $\mathrm{km}$ Gleis Io ooo Mk., unabhängig von der Bahnlänge.

2. Das Legen der Schienen nebst Pflasterung wird ebenfalls für alle 3 Systeme, unabhängig von der Bahnlänge, dasselbe kosten und zwar für das $\mathrm{km}$ Gleis $56000 \mathrm{Mk}$.

Bei Aufreissen des vorhandenen Pflasters unter Wiederverwendung desselben für das $\mathrm{km} 6250 \mathrm{Mk}$.

3. Hochleitung und Tiefleitung.

$O$. Eisenmaste, Rollendraht, Aufhängungsmaterial und Arbeitslohn (U. St. of Amerika) für das $\mathrm{km}$ $6250 \mathrm{Mk}$. eingleisig und 1 i $250 \mathrm{Mk}$. zweigleisig.

$U$. Bei Kanalsystem variieren die Kosten ganz bedeutend, werden aber immer höher als $O$ sein. Schätzungsweise kosten das $\mathrm{km}$ I 9 ooo $\mathrm{Mk}$, eingleisig und $38000 \mathrm{Mk}$. zweigleisig.

A. Hier fallen die Kosten unter 3. $O$ und 3. $U$ vollstăndig fort.

4. Speiseleitung und deren Montage.

o. $U$. Die Dicke der Speiseleitung richtet sich nach der Länge der Bahn. (Tabelle I giebt für verschiedene Bahnlänge und Wagenanzahl Preise an.)

A. Benötigt keiner Speiseleitungen. 